\title{
Optimum Power Allocation and Bit Loading with Code Rate Constraints
}

\author{
David Matas, Meritxell Lamarca \\ Signal Theory and Communications Department, Technical University of Catalonia \\ Barcelona, Spain \\ Email: (dmatas, xell)@gps.tsc.upc.edu
}

\begin{abstract}
In this paper, a new power allocation and bit loading policy is defined for those systems working with a preselected binary channel code and specific bit error rate (BER) requirements. It consists on the maximization of the spectral efficiency with a constraint on the average mutual information per coded bit (bit MI), exploiting the relationship of the bit MI with the BER and the code rate.

An irregular modulation approach is employed in order to express the policy as a convex optimization problem, solved without the need of greedy algorithms. Results are compared with those obtained with other algorithms in the literature.
\end{abstract}

\section{INTRODUCTION}

In slow fading wireless communications, channel variations can be accurately tracked by both transmitter and receiver. This enables the use of adaptive schemes in which the transmitter parameters are adjusted to optimize the performance according to the instantaneous channel response.

For systems modeled as a set of parallels channels (e.g. OFDM without intersymbol interference), the design of an adaptive transmission involves the allocation of power and bits to each one of these subchannels.

The most well-known criterion for resource allocation in parallel channels is the maximization of the channel capacity under an average power constraint. This leads to the use of Gaussian symbols and to the classical waterfilling power allocation policy [1, Sec 10.4]. However, practical communication schemes employ a limited number of discrete constellations and channel codes, so the bit rate assignments are constrained to belong to a discrete set with limited granularity defined by the size of these constellations and the rates of the codes. In this case, the waterfilling power allocation policy is not guaranteed to provide the best performance and, furthermore, a bit loading policy needs to be defined.

If the bit loading is already established, i.e. the constellation to be used on each subchannel is fixed, the optimum power allocation for the input-output mutual information (MI) maximization is mercury/waterfilling policy (MWF) [2]. However, this policy does not guarantee a good performance in a practical system where the channel code rate is fixed. Indeed, while MWF optimizes the mutual information per channel access (i.e. per transmitted symbol), the performance of the

This work has been partially funded by the Spanish/Catalan Science and Technology Commissions and by FEDER funds from the European Commission: TEC2007-68094-C02-02, 2005SGR-00639. practical system depends on whether the mutual information per coded bit (bit MI) is above/below the binary code rate. For example, although QPSK and 16QAM provide a mutual information of 1 bit per channel use in the AWGN channel at $\mathrm{SNR}=0 \mathrm{~dB}$ and, therefore, they provide equivalent solutions from the point of view of the MI per channel access, if the code rate was fixed to 0.4 then QPSK would grant reliable transmission at this SNR while 16QAM would not.

In this paper we propose an optimum joint power allocation and bit loading policy for coded modulation schemes where the forward error correction (FEC) stage consists of a predetermined capacity-approaching binary code. The design is based on imposing constraints on the per-bit mutual information, which has been shown to be a good indicator of the coded BER performance for capacity-approaching codes [4]. Hence, if we guarantee a minimum bit MI we are also guaranteeing the BER to be below a certain threshold (in fact, for an ideal capacity-achieving code the BER would be zero if the bit MI was larger than the code rate).

The proposed criterion consists on the maximization of the spectral efficiency with power and channel code rate constraints. It is expressed as a convex optimization problem making use of an irregular modulation formulation [5], and can be applied for any scheme operating on a set of parallel channels. The proposed convex approach is similar to the one in [8], but the introduction of the code rate constraints leads to rather different conclusions.

\section{IRREGULAR MODULATION AND POWER APPROACH}

Irregular modulation was first proposed in [5] and consists on the use of different constellations within a single channel. This idea has been extended to irregular modulation and power in various works for the design of practical bit loading schemes that take the code performance into account [6][7]. In this section we define the parameters and metrics that characterize this scheme.

Consider the transmission of a binary encoded stream through $Q$ independent and parallel subchannels with gains $\left\{H_{1}, \ldots, H_{Q}\right\}$. The received signal is

$$
y_{q}=H_{q} \sqrt{p_{q}} x_{q}+n_{q} \quad q=1, \ldots, Q
$$

where $p_{q}$ is the allocated power and $x_{q}$ the unit-power transmitted symbol, which belongs to one of the $N$ avail- 
able constellations, $\left\{\mathcal{C}_{1}, \ldots, \mathcal{C}_{N}\right\}$, with $\left\{m_{1}, \cdots, m_{N}\right\}$ bits per symbol respectively. The term $n_{q}$ is the additive complex white Gaussian noise (AWGN) of zero mean and variance $\sigma^{2}$.

Following an irregular modulation and power scheme, we allow the transmission of symbols belonging to different constellations with different allocated power within the same subchannel. Let $\alpha_{i q}$ be the fraction of symbols transmitted through the $q$-th subchannel which belong to the constellation $\mathcal{C}_{i}$, and $p_{i q}$ be the power allocated to each one of them. According to their definition, these parameters must fulfill:

$$
\alpha_{i q} \geq 0, \quad p_{i q} \geq 0 \quad \text { and } \quad \sum_{i=1}^{N} \alpha_{i q} \leq 1
$$

for $q=1, \ldots, Q$ and $i=1, \ldots, N$. If $\alpha_{i q}=0$, then constellation $\mathcal{C}_{i}$ is not employed in the $q$-th channel. Note also that this formulation can be mathematically seen as a continuous relaxation of the usual bit allocation, in which $\alpha_{i q} \in\{0,1\}$ (i.e. only one constellation can be used per subchannel).

Using the defined parameters, the power allocated to and the MI of the $q$-th subchannel are obtained as the weighted average of the individual values for each constellation,

$$
\begin{gathered}
P_{q}=\sum_{i=1}^{N} \alpha_{i q} p_{i q} \\
\bar{I}_{q}=\sum_{i=1}^{N} \alpha_{i q} I_{i}\left(p_{i q} \gamma_{q}\right)
\end{gathered}
$$

where $\gamma_{q}=\left|H_{q}\right|^{2} / \sigma^{2}$ is a measure of the strength of the subchannel and $I_{i}(\rho)$ is the MI between the transmitted symbols and the corresponding observations at the subchannel output for the $i$-th constellation when it is employed in an AWGN channel with SNR $\rho$.

The total MI averaged over all subchannels is

$$
\bar{I}=\frac{1}{Q} \sum_{q=1}^{Q} \bar{I}_{q}
$$

The spectral efficiency, defined as the average number of coded bits transmitted per channel access ${ }^{1}$, can be expressed as

$$
\eta=\frac{1}{Q} \sum_{q=1}^{Q} \sum_{i=1}^{N} \alpha_{i q} m_{i}
$$

Finally, the average MI per bit is obtained as:

$$
\bar{R}=\frac{\bar{I}}{\eta}
$$

\section{PROBLEM STATEMENT}

The proposed power allocation and bit loading strategy maximizes the spectral efficiency in systems where a fixed binary channel code is employed and a certain BER performance is

\footnotetext{
${ }^{1}$ Note that spectral efficiency usually refers to information bits. However, if the binary channel code rate is fixed, optimizing the system using any of the two definitions is equivalent.
}

required. In order to do so we jointly design these stages to guarantee a minimum MI per bit $R_{*}$, i.e.

$$
\bar{R} \geq R_{*}
$$

As indicated in the introduction, the minimum bit MI requirement guarantees the BER will be below the desired threshold. Note also that if the code were ideal then $R_{*}$ would be precisely the code rate.

The power allocation and bit loading that maximizes the spectral efficiency, defined in equation (6), with average power and binary code rate constraints can be expressed using equations (7) and (8) as the solution to the following optimization problem:

$$
\begin{gathered}
\max _{\alpha_{i q}, p_{i q}} \sum_{q=1}^{Q} \sum_{i=1}^{N} \alpha_{i q} m_{i} \\
\text { s.t. } \quad \alpha_{i q} \geq 0 \quad p_{i q} \geq 0, \quad \forall i, q \\
\sum_{i=1}^{N} \alpha_{i q} \leq 1 \quad \forall q \\
\frac{1}{Q} \sum_{q=1}^{Q} \sum_{i=1}^{N} \alpha_{i q} p_{i q}=P_{T} \\
\frac{1}{Q} \sum_{q=1}^{Q} \sum_{i=1}^{N} \alpha_{i q}\left(I_{i}\left(p_{i q} \gamma_{q}\right)-m_{i} R_{*}\right) \geq 0
\end{gathered}
$$

where $P_{T}$ is the total available power.

Let us define the following functions in order to simplify subsequent equations:

$$
J_{i}(\rho)=I_{i}(\rho)-m_{i} R_{*}
$$

They are the mutual information curves of the different constellations translated in the ordinates by a negative magnitude that depends on the its size (bits per symbol) and the code rate. Figure 1 depicts these functions for QPSK, 16QAM and 64QAM constellations and $R_{*}=0.7$.

It can be shown that the optimization problem (9) can be solved in two steps. The first step, whose complete expression is found in next section, consists on optimizing the spectral efficiency for a single AWGN channel, i.e.,

$$
\max _{\left\{p_{i}\right\},\left\{\alpha_{i}\right\}} \sum_{i=1}^{N} \alpha_{i} m_{i}
$$

with a fixed average power $P$

$$
\sum_{i=1}^{N} \alpha_{i} p_{i}=P
$$

and a fixed value $J$ for the translated MI

$$
\sum_{i=1}^{N} \alpha_{i} J_{i}\left(p_{i} \gamma\right) \geq J
$$

Denote as $\eta_{o}(J, P \gamma)$ the optimized value of the spectral efficiency (11) obtained in this case. The second step resorts to this function to maximize the global efficiency. Let $P_{q}$ and 
$J_{q}$ be the power and the translated MI allocated to the $q$-th subchannel. Then, equation (9) can be expressed as

$$
\begin{gathered}
\max _{P_{q}, J_{q}} \sum_{q=1}^{Q} \eta_{o}\left(J_{q}, P_{q} \gamma_{q}\right) \\
\text { s.t. } \quad P_{q} \geq 0 \quad \forall q \\
\frac{1}{Q} \sum_{q=1}^{Q} P_{q} \leq P_{T} \\
\sum_{q=1}^{Q} J_{q} \geq 0
\end{gathered}
$$

On the basis of this procedure, we first study the solution for the single AWGN channel with arbitrary $J$ requirements (first step) in section IV. Afterwards, we employ this result to find the solution to the multiple parallel channels case (second step) in section V.

\section{FIRST OPTIMIZATION STEP}

In this section we study the solution to the first optimization step. It consists on the design of the irregular modulation that maximizes the spectral efficiency for a single AWGN channel with an SNR $\mu$ and a generic requirement on the average translated MI function $J$. Note that if we wished to design the irregular modulation and power allocation for a single AWGN channel we should solve this step employing $J=0$.

\section{A. Formulation}

Adding the constraints on the parameters (2) to equations (11),(12) and (13) and after some manipulations, the following optimization problem remains:

$$
\begin{gathered}
\eta_{o}(J, \mu)=\max _{\left\{p_{i}^{\prime}\right\},\left\{\alpha_{i}\right\}} \sum_{i=1}^{N} \alpha_{i} m_{i} \\
\text { s.t. } \quad \alpha_{i} \geq 0, \quad p_{i}^{\prime} \geq 0, \quad i=1, \ldots, N \\
\sum_{i=1}^{N} \alpha_{i} \leq 1 \\
\sum_{i=1}^{N} \alpha_{i} p_{i}^{\prime}=1 \\
\sum_{i=1}^{N} \alpha_{i} J_{i}\left(p_{i}^{\prime} \mu\right) \geq J
\end{gathered}
$$

where the normalized parameters $p_{i}^{\prime}=\frac{p_{i}}{P}$ have been introduced in order to express the solution (i.e. $\alpha_{i}, p_{i}^{\prime}$ and $\eta_{o}$ ) as a function only of the SNR $\mu=P \gamma$ and the translated MI $J$.

\section{B. Solution}

Let us assume that the available constellations are ordered such that

$$
m_{1}<m_{2}<\ldots<m_{N}
$$

For the purpose of obtaining a uniform notation, we introduce also the null constellation $\mathcal{C}_{0}$ as the one with $m_{0}=0$ bits per symbol and mutual information $I_{i}=0$ for all SNRs.

As it happened in [8], thanks to the introduction of a change of variables $s_{i}=\alpha_{i} p_{i}^{\prime}$, the optimization in (15) can be formulated in terms of $s_{i}, \alpha_{i}$ as a convex problem, and the
KKT conditions [9] can be applied in order to solve it. After some cumbersome manipulations of KKT conditions it can be seen that, as opposed to [8], now the solution has no closedform expression (although it can still be easily described, as indicated next) and, furthermore, now irregular modulation plays a very important role, since the optimum efficiency is achieved at all SNR's except for very extreme ones combining adjacent constellations, according to the order established in (16). The irregular modulation allows the system to satisfy the bit MI constraint in (15e) with equality, so the resources are not wasted providing BER beyond the requirements and they can be allocated instead to maximize the spectral efficiency.

The solution can be obtained as follows (proof omitted). Define as $T_{i}$ the convex envelope of functions $J_{i}$ and $J_{i+1}$ for $i=1, \ldots, N-1$ (i.e. the union of both curves through their tangent). Define also $T_{0}$ as the union of $J_{1}$ and its tangent line that crosses the origin. Consider the optimization of spectral efficiency for a certain SNR $\mu$ and a certain bit MI requirement $J$. Then:

- If $J=J_{i}(\mu)=T_{i}(\mu), i=1, \ldots, N-1$, then only constellation $\mathcal{C}_{i}$ is employed in all channel uses (i.e. $\left.\alpha_{i}=1, p_{i}^{\prime}=1\right)$ and equation (15e) is satisfied with equality.

- If $J<J_{N}(\mu)$ then only constellation $\mathcal{C}_{N}$ is employed in all channel uses (i.e. $\alpha_{N}=1, p_{N}^{\prime}=1$ ) and equation $(15 \mathrm{e})$ is satisfied with inequality.

- If $T_{i}(\mu)>J>J_{i+1}(\mu)$ for $i=0, \ldots, N-1$ then constellations $\mathcal{C}_{i}$ and $\mathcal{C}_{i+1}$ are employed in all channel uses and equation (15e) is satisfied with equality. To find the optimum parameters we first find the unique solution to the equation

$$
\frac{J-J_{i}\left(D_{i}^{-1}(\rho)\right)}{\mu-D_{i}^{-1}(\rho)}=\frac{J_{i+1}\left(D_{i+1}^{-1}(\rho)\right)-J}{D_{i+1}^{-1}(\rho)-\mu}
$$

where $D_{i}$ stands for the derivative of mutual information for the $i$-th constellation w.r.t. the SNR.

Once the value of the variable $\rho$ is found we compute the parameters $\alpha_{i}, \alpha_{i+1}, p_{i}^{\prime}$ and $p_{i+1}^{\prime}$ as

$$
\begin{cases}p_{i}^{\prime}=\frac{1}{\mu} D_{i}^{-1}\left(\frac{\rho}{\mu}\right), & p_{i+1}^{\prime}=\frac{1}{\mu} D_{i+1}^{-1}\left(\frac{\rho}{\mu}\right) \\ \alpha_{i+1}=\frac{1-p_{i}}{p_{i+1}-p_{i}}, & \alpha_{i}=1-\alpha_{i+1}\end{cases}
$$

Note that the case $i=0$ is included, assuming $J_{0}(\mu)=0$ and $D_{0}(\mu)=0$. Then, only the smaller constellation $\mathcal{C}_{1}$ is employed in some channel uses (since $\alpha_{1}<1$ and $\mathcal{C}_{0}$ does not correspond to a real constellation).

- If $J>T_{0}(\mu)$ then the problem has no solution and $\eta_{o}=0$.

Figure 1 depicts the regions of the plane $(J, \mu)$ corresponding to each solution for an example with QPSK, 16QAM and 64QAM constellations and $R_{*}=0.7$. 


\section{Analysis}

The optimum efficiency $\eta_{o}$ for all values of $J$ and $\mu$ is obtained replacing in equation in (6) the $\alpha_{i}$ parameters found according to the solution provided in the previous section. Figure 2 depicts it for an specific example with $R_{*}=0.7$ and constellations QPSK, 16QAM and 64QAM. Besides, figure 3 depicts the section of this optimum efficiency for $J=0$. Note that, thanks to irregular modulation, the spectral efficiency grows smoothly with SNR. In order to evidence the benefits of irregular modulation this figure also depicts the optimum value that would be obtained if only integer bit loading $\left(\alpha_{i} \in\{0,1\}\right)$ was permitted.

The result of the first optimization stage is the set of optimum $\left(\alpha_{i}, p_{i}\right)$ values for all $(J, \mu)$ possible pairs. Note that this result depends only on the set of available constellations and the code rate constraint $R_{*}$, i.e. it does not depend on the channel realization. Since these parameters are fixed, this first optimization stage can be precomputed once and stored for further consultation for different channel realizations. Note also that the irregular modulation is embedded in this first optimization stage and, as shown in the next section, it does not increase the complexity of power allocation and bit loading over parallel subchannels.

\section{MULTIPLE PARALLEL CHANNELS}

In this section we find the solution to the problem in equations (9) exploiting the pre-computed function $\eta_{o}$. Introducing in (9) the optimum solution for the AWGN channel obtained before we obtain a problem that is now transparent to irregular modulation and has two degrees of freedom: the allocation of power $P_{q}$ and bit MI requirement $J_{q}$ for each subchannel $(q=1, \ldots, Q)$. Hence, the problem is finally re-stated as indicated in equations (14).

\section{A. Formulation}

Since the function $\eta_{o}$ is concave, the KKT conditions can be applied again to the optimization problem (14), obtaining that

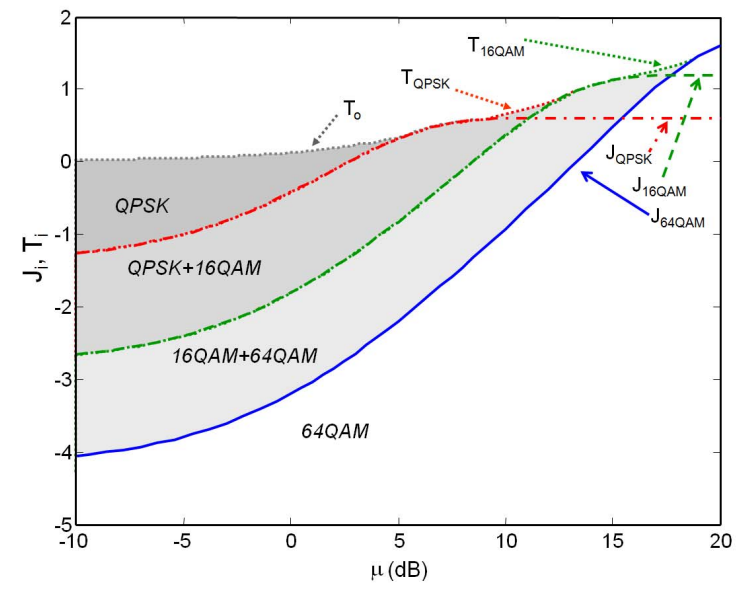

Figure 1. Translated MI as a function of the SNR $(\mu)$ for QPSK, 16QAM and 64QAM constellations and $R_{*}=0.7$. The regions where the optimum solution combines the available constellations are represented too.

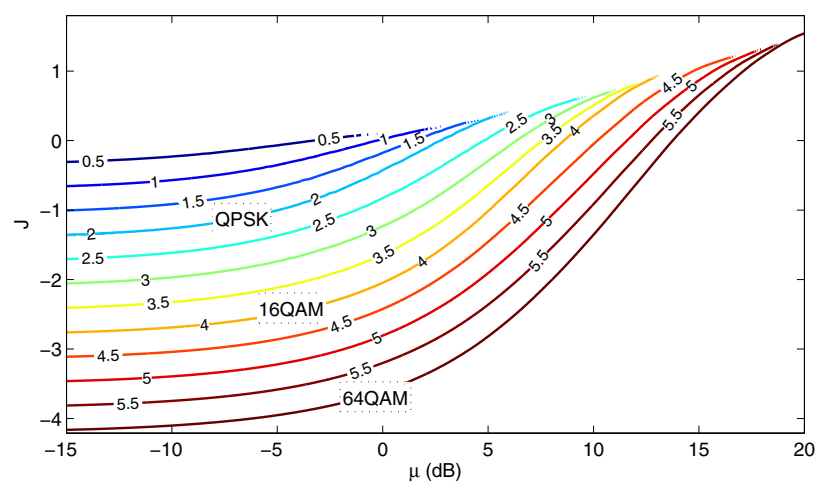

Figure 2. Contour plot of the optimum efficiency $-\eta_{o}(J, \mu)$ in equation (15a) - for an AWGN channel as a function of the SNR and the translated MI

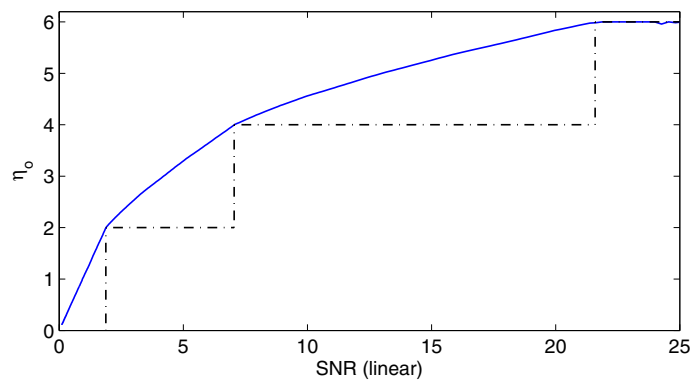

Figure 3. Section of $\eta_{o}(J, \mu)$ represented in figure 2 for $\mathrm{J}=0$ as a function of the SNR. The dashed line represents the optimum efficiency that can be achieved by integer bit loading while satisfying the bit MI constraint.

there must exist two constants $\lambda_{o}, \tau_{o}$ such that the solution satisfies:

$$
\lambda_{0}=\left.Q \gamma_{q} \frac{d \eta_{o}(J, \mu)}{d \mu}\right|_{\left(J_{q}, P_{q} \gamma_{q}\right)}, \quad \tau_{0}=\left.\frac{d \eta_{o}(J, \mu)}{d J}\right|_{\left(J_{q}, P_{q} \gamma_{q}\right)}
$$

for all used subchannels (i.e. those with $P_{q}>0$ ), all together with the power and translated MI constraints in (14). Therefore, we can express the solution (19) as

$$
P_{q}=\frac{1}{\gamma_{q}} \nabla_{P}^{-1} \eta\left(\tau_{0}, \frac{\lambda_{0}}{Q \gamma_{q}}\right), \quad J_{q}=\nabla_{J}^{-1} \eta\left(\tau_{0}, \frac{\lambda_{0}}{Q \gamma_{q}}\right)
$$

with values $\lambda_{o}, \tau_{o}$ selected to guarantee that the constraints are fulfilled.

The solution is found then in an analogous manner than in the waterfilling algorithm, but now we must perform a twodimensional search among the two waterlevels $\left(\lambda_{0}, \tau_{0}\right)$, what implies a complexity increase. This complexity increase, is due to the presence of a new dimension (the translated MI), which is in turn consequence of the new code rate constraint.

\section{B. Simulation results}

In this section we illustrate the performance of the proposed algorithm with an off-the-shelf LDPC code. Similar results would be obtained if an ideal capacity-achieving code (i.e. BER=0 with $R_{*}$ being the code rate) was considered.

Let us consider a regular $(3,6)$ LDPC code (i.e. rate 0.5 ) with a codeword length of $10^{5}$ bits. Figure 4 depicts the BER of this code with QPSK as a function of the channel 
SNR and as a function of the per-bit MI between the coded bits and the channel output. Let us assume that we wish to design a system capable of operating at a target BER of $10^{-4}$. Hence, this code would require an SNR of $1.22 \mathrm{~dB}$ or, equivalently, a bit MI of $R_{*}=0.58$ to achieve this performance. We have employed the proposed algorithm to design the bit loading and power allocation for a system using this LDPC code to transmit in an scenario with five parallel subchannels with gains $[0.45,0.25,0.15,0.1,0.05]$. Besides, we have compared the performance of our algorithm with two additional bit/power allocation policies in the literature. The first one is the Levin Campello (indicated as 'LC') bit loading algorithm [3], which is based on the Gaussian capacity with the SNR gap approximation ( this gap is $1.22 \mathrm{~dB}$ in the example scenario, as can be appreciated from figure 4). The second algorithm is the one proposed by $\mathrm{Yan} \mathrm{Li}$ (indicated as ' $\mathrm{Li}$ ') in [4] which relies on the same mutual information criterion adopted here, being the bit MI constraint $R_{*}=0.58$ too. For both them, in the simulations, the bit MI has been further improved reallocating the power by means of MWF [2], while leaving the spectral efficiency unchanged.

Fig 5 depicts the spectral efficiency and the per-bit mutual information achieved by the bit loading and power allocation provided by the three algorithms in the proposed scenario. Note that at some SNR's the LC efficiency surpasses that of the proposed algorithm, but this is at the expense of not satisfying the bit MI requirement (i.e. having a BER larger than the desired one). The Li algorithm satisfies the bit MI requirement at all SNR's, but its spectral efficiency performance is always worse than the proposed algorithm because the bit MI surpasses at large the 0.58 required value in most SNR's.

The LC and Li algorithms exhibit an step-wise form of the efficiency curve. This behavior would be less evident if the number of parallel channel were increased. Nevertheless, it could be observed that the loss of these algorithms with respect to the one proposed in this paper would remain similar. This is because power is allocated in discrete amounts in other bit loading policies (power allocation is optimized separately) and also because the use of irregular modulation allows to achieve non-integer bit loading and to verify the BER requirement tightly. Note also that these two algorithms are greedy, so the discrete bit loading and power granularity are inherent to them and guarantee their low-complexity implementation. However, in the proposed algorithm the low complexity arises from the use of a two-dimensional waterlevel search that only depends on the power and bit MI allocated per subchannel and is transparent to the bit-loading policy, which is obtained by a look-up table once the waterlevels have been determined.

\section{CONClusions}

In this paper we have obtained a new power allocation and bit loading policy for the maximization of the spectral efficiency with power and code rate constraints. The code rate constraint consists on the requirement of a lower bound on the average mutual information per bit. This criterion is expressed

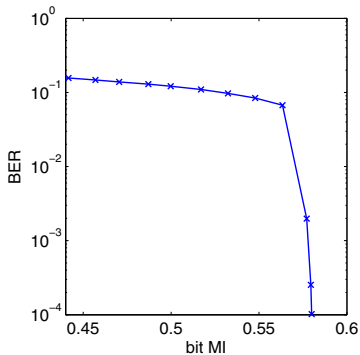

(a) BER vs MI

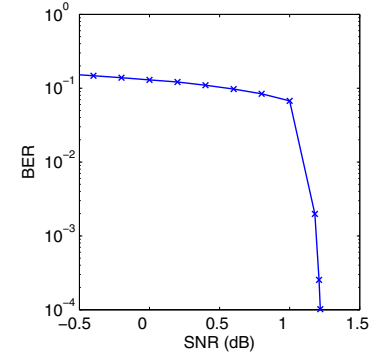

(b) BER vs SNR
Figure 4. BER as a function of the mutual information per coded bit at the channel output (a) and the SNR (b) at the input of an $r=1 / 2$ LDPC decoder (codeword of 50000 bits).

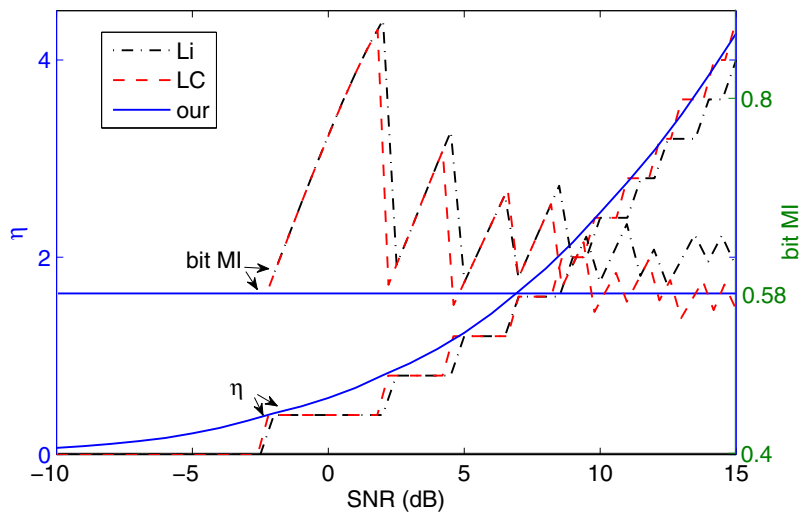

Figure 5. Optimum efficiency $(\eta)$ and bit mutual information (bit MI) resulting from our algorithm and the $\mathrm{LC}$ and $\mathrm{Yi}$ ones for the scenario described in section $\mathrm{V}$-B.

as a convex optimization problem and is found to be equivalent to a two-step optimization. For the first step, a simple description is obtained for an equivalent efficiency function for a single Gaussian channel, which can be precomputed for the desired code rate. Then, the second step employs this function to define a two-dimensional 'waterfilling' algorithm among the multiple parallel channels.

\section{REFERENCES}

[1] T. M. Cover and J. A. Thomas, Elements of Information Theory, Wiley, New York, 1990

[2] A.M. Tulino A. Lozano and S. Verdu, "Optimum power allocation for parallel gaussian channels with arbitrary input distributions," IEEE Trans. on Information Theory, vol. 52, pp. 3033- 3051, July 2006.

[3] J. Campello, "Practical bit loading for DMT," Proc. Int. Conf. Communications (ICC'99), Vancouver, June 1999, vol. 2, pp. 801- 805.

[4] Yan Li and William E.Ryan, "Mutual-information-based adaptive bit loading algorithms for LDPC-coded OFDM," IEEE Trans. on Wireless Communications, vol. 6, pp. 1670- 1680, May 2007.

[5] G.Bauch F.Schreckenbach, "Bit-interleaved coded irregular modulation," Euro.Trans.Telecomms, 2006, pp. 269-282.

[6] O.Lahuerta and M.Lamarca, "Joint design of code, constellation and power allocation in BICM LDPC coded systems," 4th Intl. Symp. on Turbo Codes and Related Topics, 2006.

[7] D. Matas and M. Lamarca, "Bit Loading and Power Allocation for BICMID with Convolutional Codes," Signal Processing Advances in Wireless Communications (SPAWC'07), June 2007.

[8] D. Matas and M. Lamarca, "Optimum Power Allocation and Bit Loading for Systems with Suboptimum Receivers," 50th Annual Global Communications Conference (GLOBECOM'07), November 2007.

[9] S. Boyd and L. Vandenberghe, Convex Optimization, Cambridge Univ. Press, Cambridge, 2004. 